\title{
Study of Energy Efficient Images with Just Noticeable Difference Threshold Based on Feature Transform
}

\author{
Ankit Kumar Soni1, Prof. Madhuvan Dixit ${ }^{2}$ \\ 1PG Scholar, ${ }^{2}$ Assistant Professor \\ 1,2Department of CSE, Millennium Institute of Technology and Science, Bhopal, Madhya Pradesh, India
}

\begin{abstract}
How to cite this paper: Ankit Kumar Soni | Prof. Madhuvan Dixit "Study of Energy Efficient Images with Just Noticeable Difference Threshold Based on Feature Transform" Published in International Journal of Trend in Scientific Research and Development (ijtsrd), ISSN: 24566470, Volume-3 | Issue-3 , April 2019, pp.924-927, URL: https://www.ijtsrd.c om/papers/ijtsrd23 215.pdf

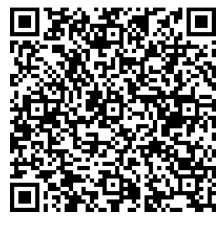

IITSRD23215

Copyright (c) 2019 by author(s) and International Journal of Trend in Scientific Research and Development Journal. This is an Open Access article distributed under the terms of the Creative Commons

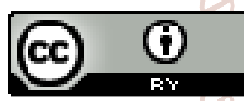
Attribution License (CC BY 4.0) (http://creativecommons.org/licenses/ by $/ 4.0$ )
\end{abstract}

\section{INTRODUCTION}

We live in an Information Technology (IT) era where computers, data centers, servers, internet and other machineries are used in almost all industries. Their goal is to assist organizations in being smart, increasing profitability and operational efficiency and increasingly to help them to become or remain sustainable in the competitive global market. From a sustainability viewpoint, however, the high usage of technology brings out several issues, such as extreme power consumption and an expanding carbon footprint that has a detrimental effect on the environment. Increasingly, these issues induce a demand for environmental sustainability concerns because of global societal concerns about energy usage, climate change, and the consequences of these changes. These concerns are important to the IT sectors because high usage of technology does contribute to the increase of greenhouse gas emissions. In response to these challenges, the term Green IT has been coined with reference to initiatives that are focused on reshaping IT into environmentally friendly forms.

Digital Image processing is part of daily computations. The graphics processing unit (GPU) has become an essential part of today's conventional computing systems. In few years, there has been a marked raise in the performance and capabilities of GPUs. Graphics Processing Units is powerful, programmable, and highly parallel computing, which are increasingly targeting for general-purpose computing applications. The modern GPU is not only a powerful graphics engine but also a highly parallel programmable processor featuring peak arithmetic and memory bandwidth that substantial as CPU. The GPU's has capability computing broad range of computation, complex problems solve \& especially for high range of graphical data. This effort in general purpose computing on the GPU, also known as GPU computing, which proposed GPU as an alternative to traditional microprocessors in high-performance computer systems of the future. The Graphics processing units (GPUs) are being increasingly used to accelerate a wide variety of applications. Recent advances in GPU architectures have not only improved the baseline performance but have also provided programmers with new options to optimize their applications for better performance. Despite these advances, writing efficient GPU applications remains a challenging task. Chief among these challenges is the problem of memory performance. GPGPU refers to the use of graphics processing units (GPUs) to perform general processing tasks, and it involves explicitly copying large amounts of data over the PCIs bus, between the CPU and GPU. This process can be 
relatively time consuming. Further complications and slowdowns can occur if the data does not fit on the GPU's memory, multiplying the problem by necessitating frequent CPU-GPU data transfers. Unlike a CPU, a GPU lacks the benefit of automatic memory paging. As a result, GPU memory management is explicitly handled by the programmer. This makes memory optimization a delicate process. The large number of threads running on a GPU will multiply any mistakes and inefficiencies in the code. This can seriously hamper an application's performance. Differences in algorithms and GPU architectures serve to complicate this matter even further. Data that arrives on a GPU's memory must then be accessed by a huge number of threads via the memory hierarchy. The threads need to be able to access this data efficiently, and through limited means. Inefficient GPU memory access is a common occurrence, and serves to hamper performance even further, particularly in workloads that are data intensive.

\section{BACKGROUND}

We propose a novel framework for performance optimization in Internet of Things (IoT)-based nextgeneration wireless sensor networks. In particular, a computationally-convenient system is presented to combat two major research problems in sensor networks. First is the conventionally-tackled resource optimization problem which triggers the drainage of battery at a faster rate within a network. Such drainage promotes inefficient resource usage thereby causing sudden death of the network. The second main bottleneck for such networks is that of data degradation. (Muzammil Behzad, Manal Abdullah, Muhammad Talal Hassan, Yao Ge and Mahmood Ashraf Khan; 2018)

Aimed at a low-energy consumption of Green Internet of Things (IoT), this paper presents an energy-efficient compressive image coding scheme, which provides compressive encoder and real-time decoder according to Compressive Sensing (CS) theory. The compressive encoder adaptively measures each image block based on the blockbased gradient field, which models the distribution of block sparse degree, and the real-time decoder linearly reconstructs each image block through a projection matrix which is learned by Minimum Mean Square Error (MMSE) criterion. (Ran Li, Xiaomeng Duan, Xu Li, Wei He and Yanling Li; 2018)

Enhancing energy/resource efficiency of neural networks is critical to support on-chip neural image processing at Internet-of-Things edge devices. This paper presents recent technology advancements towards energy-efficient neural image processing. 3D integration of image sensor and neural network improves power-efficiency with programmability and scalability. Computation energy of feed forward and recurrent neural networks is reduced by dynamic control of approximation and storage demand is reduced by imagebased adaptive weight compression. Emerging devices such as tunnel FET and Resistive Random Access Memory are utilized to achieve higher computation efficiency than CMOSbased designs. (Jong Hwan Ko, Yun Long, Mohammad Faisal Amir, Duckhwan Kim, Jaeha Kung, Taesik Na, Amit Ranjan Trivedi and Saibal Mukhopadhyay; 2017)

A novel method is presented for producing energy-efficient images, i.e., images that consume less electrical energy on energy-adaptive displays, yet have the same, or very similar perceptual quality to their original images. The proposed method relies on the fact the energy consumption of pixels in modern energy-adaptive displays like OLED displays are directly proportional to the luminance of the pixels. Hence, in this paper to reduce the energy consumption of an image while at the same time preserving its perceptual quality, it is proposed to reduce the luminance of the pixels in the image by one just-noticeable-difference (JND) threshold. (Hadi Hadizadeh; 2017)

Because of the downlink bandwidth bottleneck and power limitation on satellite, the demands for low power cost high performance on-board payload data processing which can reduce the volume of communication data are growing as well. This paper proposes high efficiency architecture for onboard hyperspectral image classification in a Zynq Soc to achieve real-time performance. (Ning Ma, Shaojun Wang, Syed Mohsin Ali, Xiuhai Cui and Yu Peng; 2016)

It is critical in terms of approximate computation errors in VLSI multiplier circuits are increasing with technology scaling. The most common method for fast and energy efficient execution of multiplication result is approximation of operands. But this traditional approximate result is not suitable for image processing applications. This paper proposes the two architectures of high accurate hybrid segment approximate multiplier (HSAM) and enhanced HSAM for image compression. (Jamuna Ramasamy and Sathishkumar Nagarajan; 2016)

Health care applications have become boon for the healthcare industry. It needs correct segmentation connected with medical images regarding correct diagnosis. An efficient method assures good quality segmentation of medical images. Segmentation methods are classified as edge based, region based, clustering based, Level set methods (LSM) and Energy based methods. In this paper, a survey on all the effective methods those are capable for accurate segmentation is given, however quick process employing correct segments is still difficult. Some existing methods do correction and some badly pertain to deep irregularity in images. The wide range of the problems of computer vision may make good use of image segmentation. (Pamela Juneja and Ramgopal Kashyap; 2016)

\section{PROBLEM IDENTIFICATION}

The distinguished issue in existing work is as per the following:

1. Color intensity is imbalance in CRT screen

2. Outdoor images may reflect high brightness in flat display.

3. Energy of images may increase in corresponding display devices.

\section{METHODOLOGY}

The method is Feature Transform based Just Noticeable Difference Threshold (FTJNDT), which is described through following point.

Step1 : Select input image I.

Step2 : The luminance channel of a given input image is first partitioned into $\mathrm{N} \times \mathrm{N}$ blocks, and then the DCT transform of all blocks are computed.

Step3 : The value of $\mathrm{N}$ is set to 8. After that, the base JND threshold for each $\mathrm{N} \times \mathrm{N}$ block is computed using the following formula: 


$$
T_{b}\left(n, i, j, w_{i j}\right)=\frac{e \exp \left(c w_{i j}\right)}{\left(\phi_{i} \phi_{j}\right)\left(a+b w_{i j}\right)\left(d+(1-d) \cos ^{2} \psi_{i j}\right)}
$$

where $\mathrm{i}, \mathrm{j}$ are the horizontal and vertical indices of the DCT coefficient at location $(\mathrm{i} ; \mathrm{j})$ for $\mathrm{i} ; \mathrm{j}=0, \ldots \ldots . ., \mathrm{N}-1$ and $\mathrm{a}=1.33, \mathrm{~b}$ $=0.11, \mathrm{c}=0.18, \mathrm{~d}=0.6, \mathrm{e}=0.25$. Also, $\phi_{i}$ and $\phi_{j}$ are defined as:

$$
\phi_{m}=\left\{\begin{array}{c}
\sqrt{\frac{1}{N}}, m=0 \\
\sqrt{\frac{2}{N}}, m>0,(m=i, j)
\end{array} .\right.
$$

and the spatial frequency of the (i, j)-th DCT coefficient is defined as $\omega_{i j}=\frac{1}{2 N} \sqrt{\left(\frac{i}{\theta_{x}}\right)^{2}+\left(\frac{j}{\theta_{y}}\right)^{2}}$, where $\theta_{x}$ and $\theta_{y}$ are the horizontal and vertical visual angles of a pixel, and they can be calculated using the following equation based on the viewing distance $l$ and the actual width and height of a pixel on the monitor, Px and Py:

$$
\theta_{t}=2 \arctan \left(\frac{P_{t}}{2 l}\right),(t=x, y)
$$

Also, $\psi_{i j}$ in (1) denotes the directional angle of the $(\mathrm{i}, \mathrm{j})$-th DCT coefficient, and it is defined as $\psi_{i j}=\arcsin \left(\frac{2 \omega_{i, 0} \omega_{0, j}}{\omega_{i j}^{2}}\right)$.

The term $1 /\left(d+(1-d) \cos ^{2} \psi_{i j}\right)$ in (1) accounts for the so called oblique effect, which models the fact that the human visual sensitivity in the horizontal and vertical directions (when $\mathrm{i}$ or $\mathrm{j}$ equals to 0 ) is higher than other directions (e.g. when $\mathrm{i}=\mathrm{j}$ ).

Step 4: Now obtain feature descriptor of luminance channel through following steps.

Scale-Space Extreme Detection: Theinitial step of evaluation finds total allscale-space and different image area in image dataset nodes. It is completely apply effectively by using a Difference-of-Gaussian (DoG) mapping to represents potential interest key points of feature descriptors which are scale invariant and orientation in image dataset nodes.

Key points Localization: All candidate area of image in selected ROI (Region of Interest), a detailed prototype is fit to analyze key points area and its scale-space. Key points of image area in image ROI are chooses basis on calculate of existing stability.

Orientation Assignment: One or more orientations task are applied to each key points area based on local image data nodes gradient directions. Each and every future image operations are implemented on image key point dataset which has been transformed relative to the applied orientation, scale, and location for each feature descriptor, hence providing invariance to these transformations in image data nodes.
Key points Descriptor: The local image gradients value are measured at the choose scale-space in the Region of Interest (ROI) around all key points in image dataset points. These are transformed into a presentation that permits for significant levels of local shape, location and orientation and changes in illumination of image dataset points.

Step 5: Now optimize feature descriptor of luminance channel. Then obtain image is energy efficient image.

\section{RESULTS AND ANALYSIS}

To evaluate the accuracy of the proposed Feature Transform based Just Noticeable Difference Threshold (FTJNDT) as compared to the adaptive SJND model using mentioned comparison methodology, a subjective analysis is performed as follows.

Table 1: Analysis of PSNR(dB) among Niu's[24], ASJND[1] and FTJNDT(Proposed)

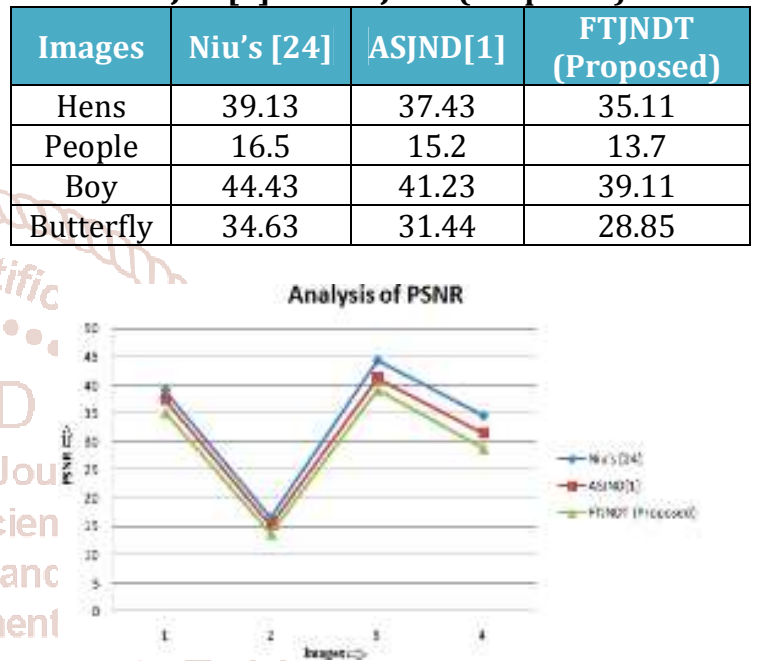

Figure 1: Graphical Comparison of PSNR(dB) among Niu's[24], ASJND[1] and FTJNDT(Proposed)

In figure 1, proposed approach reduce the PSNR as compare then Niu's[24], ASJND[1] for different image dataset. For image 1, PSNR will reduce by $6.6 \%$ as compare then ASJND[1]. Hence quality of feature transform based image has more efficient then existing work.

Table 2: Analysis of VSI (Probability of Pixel) among Niu's[24], ASJND[1] and FTJNDT(Proposed)

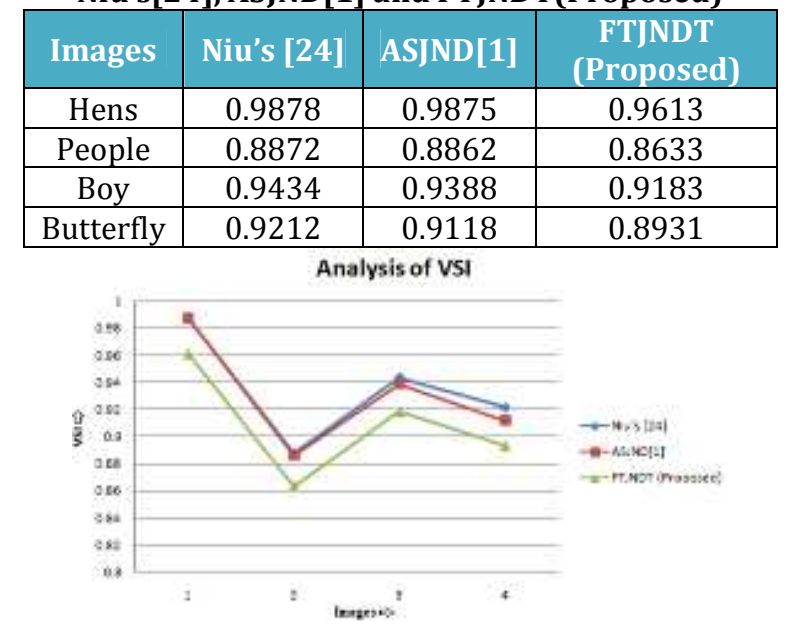

Figure 2: Graphical Comparison of VSI (Probability of Pixel) among Niu's[24], ASJND[1] and FTJNDT(Proposed) 
In figure 2, proposed approach improve the VSI as compare then Niu's[24], ASJND[1] for different image dataset. For image 1 , VSI will reduce by $2.75 \%$ as compare then ASJND[1]. Hence saliency map factor is reducing through feature transform based image which is more efficient then existing work.

Table 3: Analysis of Energy (Percentage) among
Niu's[24], ASJND[1] and FTJNDT(Proposed)
\begin{tabular}{|c|c|c|c|}
\hline Images & Niu's [24] & ASJND [1] & $\begin{array}{c}\text { FT]NDT } \\
\text { (Proposed) }\end{array}$ \\
\hline Hens & 64 & 69 & 74 \\
\hline People & 58 & 65 & 73 \\
\hline Boy & 77 & 82 & 91 \\
\hline Butterfly & 69 & 73 & 88 \\
\hline
\end{tabular}

\section{Analysis of Energy Efficiency}

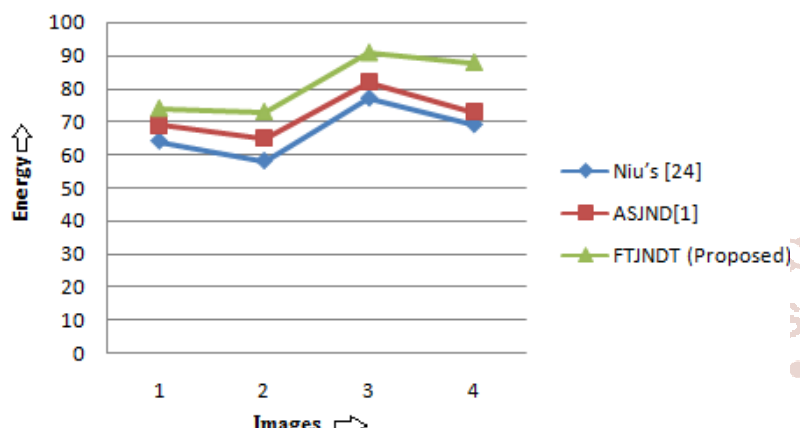

Figure 3: Graphical Comparison of Energy (Percentage) among Niu's[24], ASJND[1] and FTJNDT(Proposed)

In figure 3, proposed approach improve the Energy Efficiency as compare then Niu's[24], ASJND[1] for different image dataset. For image 1, energy will reduce by $4.31 \%$ as compare then ASJND[1]. Hence saliency map factor is reducing through feature transform based image which is more efficient then existing work.

\section{CONCLUSIONS AND FUTURE WORK}

The results obtained by real power measurements on an OLED display demonstrated that the proposed method for producing the energy-efficient images is able to reduce the energy consumption by about $4.31 \%$ on average at the same perceptual quality of the original images. This amount of saving per image can translate too many Mega Watts when all displays in the world are taken into account, which would be very considerable from the global warming point of view. The obtain conclusion of this thesis work is as per the following

1. Color intensity is improve in CRT screen upto $6 \%$

2. Outdoor images maintain brightness in flat display with VSI factor up to $2.75 \%$.

3. Energy of images is maintained up to $5 \%$.

There are many intriguing questions that future work should consider. First, our image coding scheme should be deployed into an actual hardware platform. By the energy consumption of our scheme on this hardware platform, we are to verify the trade-off among the number of CS measurements, energy consumption, and the reconstruction quality. Second, we should analyze the trade-off on energy between local codec and transmission. Importantly, by some simulation results, we hope to construct an empirical model to measure this trade-off in the future work.

\section{REFERENCES}

[1] Muzammil Behzad, Manal Abdullah, Muhammad Talal Hassan, Yao Ge and Mahmood Ashraf Khan, "Toward Performance Optimization in IoT-based Next-Gen Wireless Sensor Networks", IEEE Conference on IoT, 2018.

[2] Ran Li, Xiaomeng Duan, Xu Li, Wei He and Yanling Li, "An Energy-Efficient Compressive Image Coding for Green Internet of Things (IoT)", MDPI Journal on Sensors, 2018

[3] Jong Hwan Ko, Yun Long, Mohammad Faisal Amir, Duckhwan Kim, Jaeha Kung, Taesik Na, Amit Ranjan Trivedi and Saibal Mukhopadhyay, "Energy-Efficient Neural Image Processing for Internet-of-Things Edge Devices", IEEE Transaction on IoT, 2017.

[4] Hadi Hadizadeh, "Energy-efficient Images", IEEE Transactions on Image Processing, 2017.

[5] Ning Ma, Shaojun Wang, Syed Mohsin Ali, Xiuhai Cui and Yu Peng "High Efficiency On-Board Hyperspectral Image Classification with Zynq SoC", MATEC Web of Conference, 2016.

[6] Jamuna Ramasamy, Sathishkumar Nagarajan, "Hybrid Segment Approximate Multiplication for Image Processing Applications", Circuits and Systems, 2016.

[7] Pamela Juneja and Ramgopal Kashyap, "Energy based Methods for Medical Image Segmentation", International Journal of Computer Applications (0975 8887) Volume 146 No.6, July 2016.

[8] Rashmi S Mahuli and Dr. Shridhar Joshi, "Energy Efficient and Secure Image Transmission in WSN", International Journal of Innovative Research in Computer and Communication Engineering, Vol. 4, Issue 4, April 2016.

[9] Deguang Li, Bing Guo, Yan Shen, Junke Li and Yanhui Huang, "Making Image More Energy Efficient for OLED Smart Devices", Hindawi Publishing Corporation Mobile Information Systems, Volume 2016.

[10] Yao Liu, Mengbai Xiao, Ming Zhang, Xin Li, Mian Dong, Zhan Ma, Zhenhua Li and Songqing Chen, "GoCAD: GPUAssisted Online Content-Adaptive Display Power Saving for Mobile Devices in Internet Streaming", International World Wide Web Conference Committee (IW3C2), 2016.

[11] Sumant Mukherjee and Dushyant Kumar Soni, "Energy Efficient Multiplier for High Speed DSP Application", International Journal of Computer Science and Mobile Computing, Vol.4 Issue.6, June- 2015. 